\title{
The specificities of diagnostic examination of childhood Obstructive Sleep Apnea- Hypopnea Syndrome (OSAHS)
}

\author{
T. Séailles ${ }^{1}$, M.-F. Vecchierini ${ }^{2}$
}

1 Sleep physician, Multidisciplinary Functional Exploration Dept, Ambroise-Paré Hospital

2 Neurologist, Lecturer and Honorary Hospital Practitioner, Sleep and Alertness Center, Hôtel-Dieu

\begin{abstract}
The diagnosis of obstructive sleep apnea-hypopnea syndrome (OSAHS) in children depends both on daytime and nighttime symptoms which vary subjectively between individuals and on the results of nocturnal monitoring, intended to supply an objective basis and precision for diagnosis and assessment of severity. Nocturnal monitoring measures the apnea and hypopnea index (AHI): if the score is $>1.5$ per hour of sleep, diagnosis is confirmed and the score is a key to determining management. Simple respiratory polygraphy can confirm OSAHS is $77 \%$ of cases, but cannot identify upper airway resistance syndrome (UARS), where apnea and desaturating hypopnea are often absent but in which restricted inspiration leads to cortical micro-arousal. UARS is relatively frequent in children and requires full polysomnography, although this tool is not readily available. The clinician can provide critical interpretation of the results of nocturnal monitoring if he or she is aware of the various techniques and their respective diagnostic sensitivity; in the light of the individual clinical context, a patient-centered approach can thus be adopted, improving management of sleep disordered breathing.
\end{abstract}

\section{KEYWORDS}

Diagnosis, obstructive sleep apnea, children

\section{INTRODUCTION}

In children with suspected sleep disordered breathing, which may range from primary snoring to upper airway resistance syndrome (UARS) and obstructive sleep apnea-hypopnea syndrome $(\mathrm{OSAHS})^{13}$, diagnostic examinations are the key to identifying respiratory abnormality and defining the disorder. Respiratory sleep recording is essential to diagnosing OSAHS and assessing severity ${ }^{22}$. It also contributes to therapeutic decision-making, which needs to be early and effective. Diagnosis of the main etiological mechanisms, specific to children, underlying OSAHS should be systematically explored so as to enable etiological treatment. Treatment efficacy is commonly assessed by further recordings to confirm resolution or persistent disorder. The present study seeks to shed light on the methodology, strengths and weaknesses of the various sleep monitoring techniques, to help the

Address for correspondence:

Thierry Séailles

9, Avenue Charles-de-Gaule

92104 Boulogne-sur-Seine - France

Article received: 08-02-2015.

E-mail: thierry.seailles@orange.fr

This is an Open Access article distributed under the terms of the Creative Commons Attribution

License (http://creativecommons.org/licenses/by/4.0),

which permits unrestricted use, distribution, and reproduction in any medium,

provided the original work is properly cited. 
physician in diagnostic decision-making and interpretation of results.

After reminders of growth-related specificities, the identified respiratory abnormalities, the definitions of the various types of sleep disordered breathing, and the different recording apparatuses and their respective performances, a diagnostic strategy is put forward and discussed.

\section{PHYSIOLOGICAL SPECIFICITIES OF CHILD GROWTH}

The impact of growth on airway morphology and neurorespiratory physiology needs to be specified. During the first years of life, airway size and tonus, respiration, sleep structure ${ }^{24}$ and degree of adiposity evolve. Consequently, OSAHS phenotypes and the signals detected during recording likewise evolve with growth.

\section{Progressive increase in airway diameter and reduction in tonus}

In children less than 3 years of age, the notable narrowness of the airways seems to be counterbalanced by greater motor tonus ${ }^{24}$, opposing complete collapse during obstructive episodes and diminishing during paradoxical sleep. Between the ages of about 3 and 8 years, frequent severe hypertrophy of adenotonsillar lymphoid tissue may lead to episodes of sometimes complete airflow obstruction. After 8 years of age, airway diameter grows wider than that of the adenotonsillar tissue, leading to overall widening, while the tonus of muscular airway opening progressively decreases ${ }^{24}$.

\section{Evolution of respiration and respiratory rate during growth}

Respiratory rate progressively decreases from $30 / \mathrm{min}$ in infants to $12 /$ min by the end of adolescence.

\section{Evolution of corpulence over growth}

Corpulence, measured by body mass index (BMI: weight divided by height squared) progressively increases from a low-point of $15 \mathrm{~kg} / \mathrm{m} 2$ around 6 years of age to $21 \mathrm{~kg} / \mathrm{m} 2$ at 18 years. In parallel, adipose tissue develops progressively, while muscle tissue increases considerably in boys after puberty.

\section{Microstructural evolution of sleep in children}

Increased sleep stability in children reduces sleep fragmentation, notably that related to respiration. Cortical micro-arousal at the end of apnea episodes is rarer in children than adults and is not found at all in $50 \%$ of cases ${ }^{1,24}$. This relative lack of fragmentation may lead to respiratory events being underestimated if associated cortical micro-arousal is required to detect them.

Physiological specificities of young children, such as a low rate of overweight, little fragmentation of sleep and elevated airway tonus, affect the results of diagnostic examination, tending to reduce the apnea-hypopnea in$\operatorname{dex}(\mathrm{AHI})$. 


\section{INTERVIEW AND CLINICAL EXAMINATION}

\section{Clinical context and subjectivity of reported symptoms}

Sleep assessment is based on clinical symptomatology disclosed on interview. In children, it is mainly the parents, teachers and those close to the patient who report symptoms that are suggestive of diagnosis (snoring, respiratory breaks, oral breathing, daytime somnolence), sometimes worrying (attention disorder, hyperactivity, enuresis, bruxism, somnambulism), and sometimes non-specific (fatigue, social withdrawal, delayed growth). The exactitude and wealth of signs noticed and reported depends on these adults' awareness of OSAHS symptoms, individual tolerance of them, the attention paid to the child during the day and the night, preconceptions as to whether signs are benign or worrying and whether they indicate sleep disordered breathing. Taken together, these factors account for the variability and subjectivity of the information given to the physician by the parents. Exploring for history suggestive of familial OSAHS involving siblings or parents (as was the case in $46 \%$ of cases in Kim's cohort ${ }^{17}$ ) is of great diagnostic and indeed prognostic value. Identifying other risk factors (prematurity, ethnicity, asthma, allergy, smoke exposure) reinforces the probability of diagnosing sleep disordered breathing. Diagnostic questionnaires ${ }^{34,37}$ are useful for screening at-risk subjects, but prove disappointing for diagnosis, which they do not confirm with certainty.

\section{Clinical examination}

If performed methodically, rigorously and in depth, clinical, anatomic and dynamic examination provides more objective assessment than the clinical symptoms as such, and above all suggests the etiological mechanisms and probable site of obstruction. Childhood OSAHS usually involves airway narrowing, due either to constitutional morphology ${ }^{17}$, adenotonsillar hypertrophy with onset between 3 and 8 years of age $^{24}$, overweight usually developing in adolescence ${ }^{18}$, or an association of the above. The growth curve quantifies overweight or failure to thrive, which may be associated with OSAHS.

Examination comprises assessment of airway size at the nares and oropharynx, sometimes using endoscopy. Pharyngeal abnormalities are assessed on Mallampatti's scale ${ }^{17}$ and tonsillar hypertrophy on Friedman's scale ${ }^{17}$; maxillomandibular position is assessed by determining dental classes ${ }^{17}$. Oral respiration, very frequently associated with OSAHS, may be visible to inspection or identified by low tongue positioning, atypical swallowing, cervical statics and impact on cranial morphology (long-face syndrome, ogival palate), or dental implantation. Facial photographs and cranial radiographs with architectural analysis contribute to identifying morphologic bone abnormalities, which affect $93 \%$ of children with OSAHS according to $\mathrm{Kim}^{17}$. ENT and manducatory system specialists contribute their specific skills and experience to identify direct and indirect signs of airway 
narrowing. These various grounds for suspecting sleep disordered breathing, however, despite the wealth of symptoms and clinical arguments, do not suffice to confirm diagnosis ${ }^{7}$. Only sleep recording provides objective confirmation, assesses severity and distinguishes the various forms of obstructive sleep disorder.

\section{DEFINITIONS}

\section{Characteristics of respiratory events identified in children ${ }^{4}$ (fig. 1)}

Obstructive apnea: $\geq 90 \%$ reduction in airflow during two respiratory cycles, associated with persistent respiratory movements identified by thoraco-abdominal muscle mobility during apnea.

Central apnea: $\geq 90 \%$ fall in airflow during 2 respiratory cycles, without respiratory movement, leading to a 3\% drop in oxygen saturation, and cortical micro-arousal or complete awakening. Central apnea is physiological and frequent in infants and young children and is not counted in calculating the obstructive apnea-hypopnea index (OAHI).

Hypopnea: $\geq 30 \%$ fall in airflow during 2 respiratory cycles, leading either to $\geq 3 \%$ desaturation or to cortical micro-arousal or complete awakening.

RERA (Respiratory Effort Related Arousal): snoring or flattened curve for in-breath airflow in the nasal cannula for $>2$ cycles, with $<30 \%$ fall in airflow or increased partial carbon dioxide pressure $\left(\mathrm{PaCO}_{2}\right)$ as assessed by air $\mathrm{CO}_{2}$ content at end of out-breath, leading to cortical micro-arousal or complete awakening.

Oxygen desaturation: $\geq 3 \%$ fall in blood oxygen level compared to previous level.
Cortical micro-arousal: usually, accelerated EEG rhythm $>16 \mathrm{~Hz}$ for 3-15 seconds 22,33 ; after 15 seconds, awakening is complete.

According to the American Academy of Sleep Medicine (AASM) rules ${ }^{4}$, RERA scoring is optional and should not be included in the calculation of AHI but only in that of the respiratory disorder index: $\mathrm{RDI}=\mathrm{AHI}+\mathrm{RERA}$ index. (Normal values and pathological threshold are not clearly defined for the RERA index.)

$\mathrm{PaCO}_{2}>50 \mathrm{~mm} \mathrm{Hg}$ in blood or expired air for $>25 \%$ of total sleep time defines alveolar hypoventilation, usually without apnea-hypopnea or cortical micro-arousal.

\section{Sleep disordered breathing}

Sleep disordered breathing consists in respiratory changes during sleep, with increasing airway obstruction, gradual gas exchange abnormalities and respiratory micro-arousal episodes. At the more benign end of the spectrum, slightly more severe, there is UARS, and at the most severe end there is OSAHS. There is no discontinuity in increasing severity, and the borderlines between conditions are arbitrary. In the present article, "sleep disordered breathing" is a generic term 


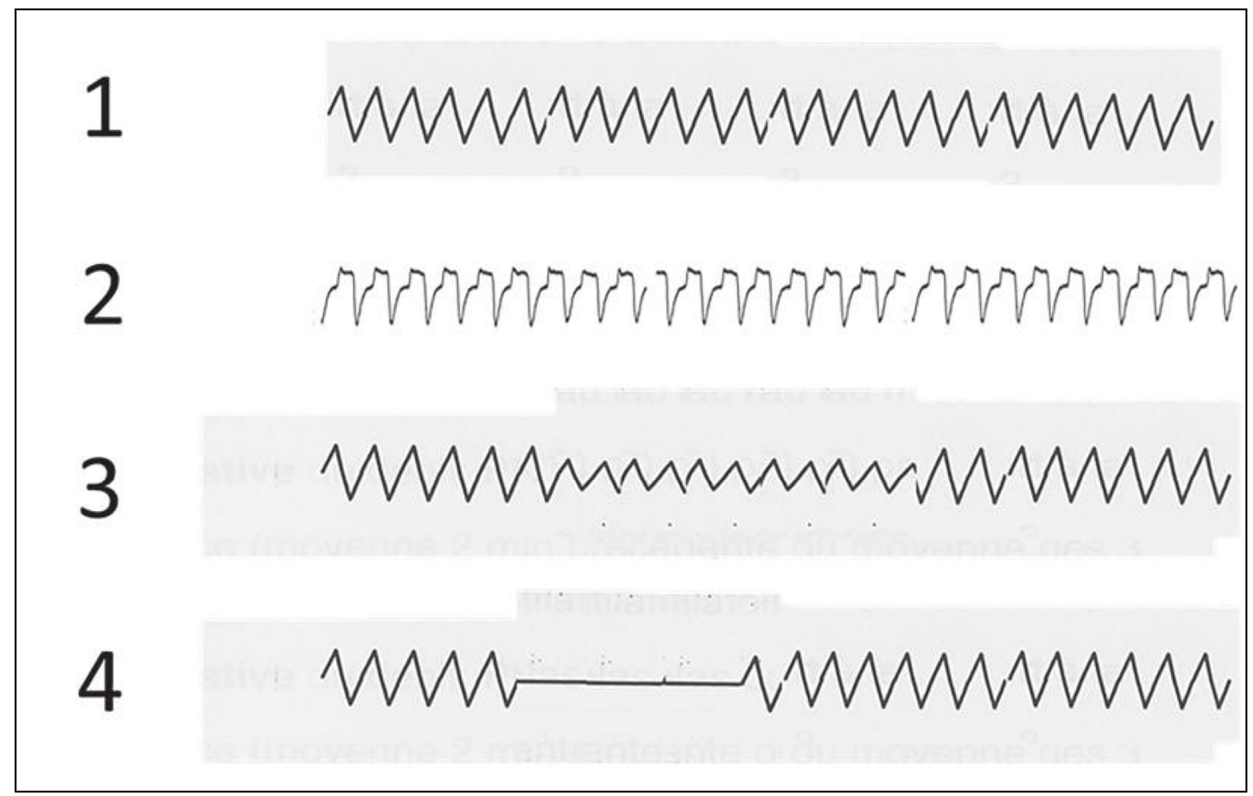

Figure 1

Respiratory abnormalities recorded in the nasal airflow cannula during sleep recording. 1: normal regular respiration with normal cycles. 2: regular breathing with in-breath restrictions seen in the plateau at the top of the respiratory curve. 3: initially normal respiration, temporarily

interrupted by hypopnea in several consecutive cycles, followed by recovery of normal amplitude. 4: initially normal respiration, temporarily interrupted by prolonged apnea for several cycles, followed by recovery of normal amplitude.

covering all obstructive sleep disorders and referring to the whole continuum, whereas primary snoring, UARS and OSAHS refer to specific categories along the continuum.

Primary or habitual snoring is defined as occurring during sleep more than 3 times per week, not usually associated with apnea or hypopnea, gas exchange disorder or cortical micro-arousal; $\mathrm{AHI}$ on PSG is $<1 / \mathrm{h}$. Primary snoring used to be considered benign, but is increasingly found to entail proven clinical morbidity 5,6,20,21,39.

UARS, first described in children by Guilleminault ${ }^{14}$, is defined as restricted inspiration accompanied by effort, leading to cortical micro-arousal known as RERA, generally without apnea, hypopnea or gas exchange disorder. The RDI combines $\mathrm{AHI}$ and RERA. Since 2005, UARS, which is not an entity in itself according to the AASM, has been subsumed under OSAHS.

OSAHS, first described in children in 1976 by Guilleminault ${ }^{12}$, features apnea or hypopnea episodes with obstructive apnea index (OAI) $>1 / \mathrm{h}$ or $\mathrm{OAHI}>$ 1.5/h. RERA may occur during OSAHS, and the RERA index can optionally be added to the $\mathrm{AHI}$.

\section{Types of sleep recording for diagnosis of OSAHS}

OSAHS diagnostic examinations are part of the diagnostic process and serve to confirm, detail and quantify respiratory 
abnormalities suspected on clinical examination by assessing the indices of certain events: apnea, hypopnea, restricted in-breath, snoring, oxygen desaturation and cortical micro-arousal.

Sleep video recording provides information on breathing during sleep; it is not enough in itself to confirm that respiratory breaks are complete or that oxygen desaturation or respiratory effort are occurring, and cannot found diagnosis.

Pulsed oximetry measures blood oxygen levels throughout the night and counts desaturation episodes equal to or greater than 3\%. Desaturation does not in itself indicate obstructive episodes or their duration. Even when oximetry reveals numerous desaturation episodes, this does not diagnose OSAHS, as they may be due to central apnea. Likewise, when oximetry reveals no desaturation, this does not rule out a diagnosis of sleep disordered breathing without desaturation. Thus oximetry neither confirms nor rules out OSAHS, and a more complete complementary examination is mandatory. A questionnaire may be associated to oximetry to enhance detection of OSAHS and improve severity assessment; such an examination can help determine an order of priority when several children require sleep recording and the waiting list is long 8 .

Simplified sleep recording by level III respiratory polygraphy (fig. 2), which the ASDA (American Sleep Disorders Association) in 1994 called "cardio-respiratory recording"31, may validate a diagnosis of OSAHS. At least 4 signals are recorded: respiratory flow, thoracic movement, heart rhythm or electrocardiogram (ECG), and pulsed oximetry to measure blood oxygen saturation. In France, the national health insurance

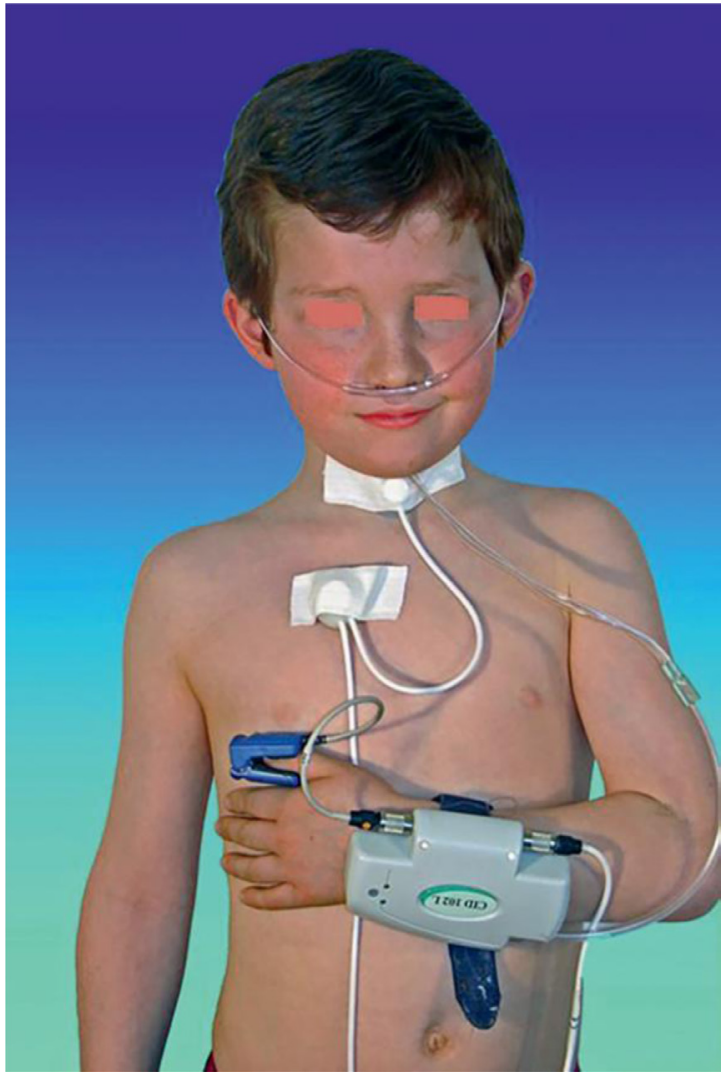

Figure 2

Type III respiratory polygraphy sensors.

(The thoracic-abdominal belts are not visible in this image.)

scheme further requires snoring and body position to be recorded. This type of examination detects all apnea episodes, both obstructive and central, but only some hypopnea episodes (according to the definitions stated above); those inducing $\geq 3 \%$ desaturation can be described while the others, associated with cortical micro-arousal, are not taken into account, as respiratory polygraphy does not include electroencephalography (EEG) electrodes to detect micro-arousal. Certain authors recommend detecting autonomic activation by pulse transit time $(\mathrm{PTT})^{16}$, pulse oximetry plethysmography and 
peripheral arterial tonometry (PAT) to describe hypopnea or RERA, but the corresponding criteria have not been adopted by the AASM.

\section{Type 2 ambulatory polysomnogra- phy $^{31}$}

Type 2 polysomnography initially used a minimum of 7 signals; at present, it comprises 3 EEG (Rolandic fissure, frontal and occipital), 2 electro-oculographic (EOG), 1 mental electromyographic (EMG) and 2 anterior tibial EMG signals plus the sensors used in respiratory polygraphy.

Certain sensors contribute to characterizing RERA by indirectly detecting respiratory effort via snoring, restricted in-breath characteristically seen in the inspiratory phase of the flow curve recorded by the nasal cannula, and phase-opposed movement in the thoracic-abdominal belts; esophageal pressure, formerly used in sleep lab recording as a proxy for the degree of negative intrathoracic pressure, is now reserved for research protocols because of its invasiveness. Optionally, diaphragm or intercostal EMG may indirectly assess respiratory effort.

These data, and especially EEG, enable analysis of sleep: duration, continuity and structure. Paradoxical or rapid-eye-movement (REM) sleep is detected by EOG and muscular atony; it is in this phase that respiratory abnormalities are the most frequent and most marked. The relation between respiratory events and sleep enables $\mathrm{AHI}$ to be calculated per phase or for sleep as a whole. Analyzing the impact of respiratory events on the continuity of sleep allows assessment of fragmentation

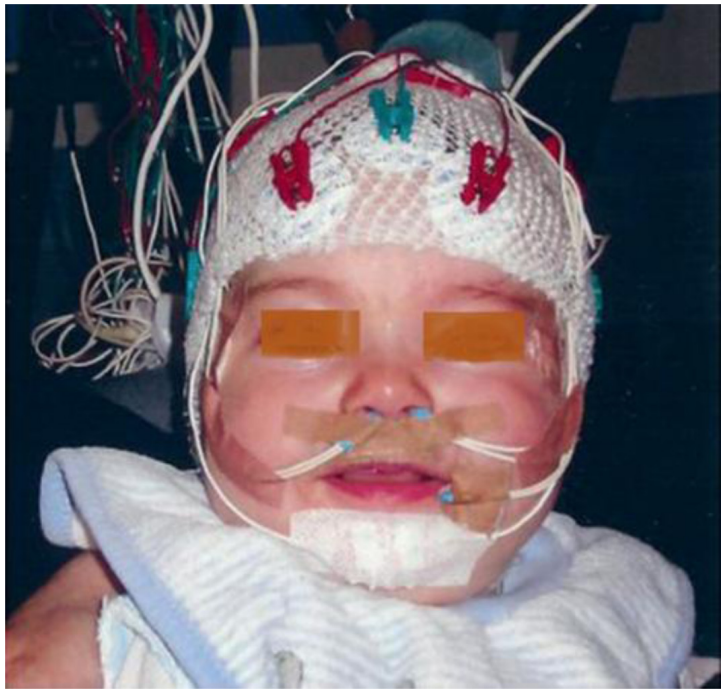

Figure 3

Cephalic polysomnography sensors.

by cortical micro-arousal and RERA. Polysomnography also allows diagnosis of other sleep pathologies such as periodic lower-limb movements, which also fragment sleep, but these are detected by anterior tibial EMG. There are also other sleep pathologies that can be diagnosed by sleep recording.

\section{Type 1 polysomnography ${ }^{31}$, in the laboratory (fig. 3)}

This examination uses the same sensors as in type-2 polysomnography, plus ECG and $\mathrm{pCO}^{2}$, measured either in the air at end of expiration or transcutaneously via a sensor on the skin. Continuous monitoring by a technician allows intervention to enhance the signal in case of sensor failure; video recording allows analysis of complex body movement. Diaphragm and intercostal EMG may also be associated. This type of polysomnography is the reference sleep examination. 


\section{DIAGNOSIS OF OSAHS}

\section{Adapting AHI diagnostic thresholds to child physiology}

Apnea-hypopnea is diagnosed by the same sleep recording sensors and apparatus in children as in adults, but with adapted criteria. Sleep structure and airway physiology in children leads to underestimation of respiratory and neurological events, explaining why studies in healthy children give obstructive apnea scores well below $1 / \mathrm{h}$; in adults, apnea scores greater than $5 / h$ are taken as diagnosing OSAHS, whereas in children a score greater than $1 / h$ is taken as threshold up to the age of 18 years $^{36}$.

\section{Influence of type of recording on diagnostic sensitivity}

Tan et al. ${ }^{35}$ studied OAHI in relation to diagnosis in 100 PSGs of children referred for suspected OSAHS, comparing respiratory polygraphy and PSG criteria. Mild OSAHS, defined in the study as AHI between 1 and 5/h, was underestimated in $27 \%$ of cases using only the sensors specific to respiratory polygraphy as compared to the full panoply of PSG. In moderate OSAHS (OAHI 5-10/h) severity was underestimated in $60 \%$ of cases. In contrast, respiratory polygraphy gave results concordant with PSG in the 20 cases of severe OSAHS (OAHI >10/h). Even so, severity was underestimated in 23 of the 100 children on respiratory polygraphy as compared to PSG.

\section{Influence of scoring rules on diagnosis}

Hypopnea scoring rules strongly impact the capacity to diagnose OSAHS in children. Comparing the 2007 AASM rules $^{15}$ to the previous 1999 system found $24 \%$ underdiagnosis in a pediatric population according to Nixon ${ }^{28}$. In 2011, Lin'22, in a group of 100 children, reported that the 2007 ASSM rules ${ }^{15}$ underestimated severity in $81 \%$ of cases compared to the Stanford rules. In 2012, the AASM hypopnea threshold was set at a $30 \%$ decrease in airflow, rather than the previous 50\%, and the diagnostic capability of sleep recording was improved. Finally, implementing optional rules for RERA scoring increased RDI scores by a mean $40-50 \%$ in adults according to ${ }^{19}$. There is thus a serious impact of scoring rules on $\mathrm{OAHI}$ values and the capacity of sleep recording to detect sleep disordered breathing.

\section{Access issues in sleep recording}

While the AASM and the American Academy of Pediatrics (AAP) recommend systematic recording ahead of adenotonsillectomy, The American Academy of Otorhinolaryngology Head and Neck Surgery recommends recording ahead of adenotonsillectomy only in case of comorbidity or discrepancy between tonsil size and clinical symptoms. The French Health Authority (HAS) has adopted the American Academy of Otorhinolaryngology - Head and 
Neck Surgery recommendations. Sleep recording is a complex, heavy procedure and waiting lists for a hospital appointment in pediatrics are especially long, sometimes several months.

\section{Diagnostic strategy}

Spryut ${ }^{34}$ and Villa ${ }^{37}$ attempted to predict diagnosis or severity from clinical findings. Actually, clinical examination cannot reliably predict diagnosis or severity, as shown by Weinstock ${ }^{38}$. Strongly positive baseline oximetry, however, founds suspicion of severe desaturating OSAHS, which may then more easily be diagnosed on respiratory polygraphy; likewise, in case of clear symptomatology revealed on a severity questionnaire ${ }^{34}$, initial respiratory polygraphy could be performed; if the result is negative, diagnosis remains undetermined and PSG will be needed. This diagnostic strategy, going from the simplest examination, which is always contributive, to a fuller examination such as respiratory polygraphy or ambulatory PSG and culminating if necessary with complete type-1 PSG, was recommended by Muzumdar ${ }^{26}$ and the American Thoracic Society (ATS) ${ }^{32}$.

\section{Special issues}

Results vary according to the complexity of sleep recording, the number and type of sensors and the scoring rules: scores and diagnosis are therefore to be interpreted in the light of the method used.

Excessive waiting times for PSG do not mean that diagnostic exploration for sleep-disordered breathing should be abandoned or that OSAHS symp- toms should be attributed to other causes deemed constitutional and irreversible: hyperactivity, attention disorder, memory impairment, anxiety, insomnia, laziness, asthenia, heredity, etc. On the contrary, restricted access to sleep recording leads to underestimation of OSAHS by both physicians and families.

\section{The problem of primary snoring with OSAHS symptoms}

There is an important problem relating to the discrepancy between recording data and clinical impact in primary snorers with OSAHS symptoms. These children present no identifiable signs on complete PSG apart from snoring and a few episodes of restricted airflow. When a group of primary snorers is compared to non-snoring controls, however, significant differences emerge: the former show attention deficit, lower cognitive ability, greater hyperactivity, more frequent enuresis, arterial hypertension ${ }^{20}$, endothelial dysfunction ${ }^{21}$, and elevated serum resistin ${ }^{39}$. All this combines to assimilate the complications experienced by primary snorers to those typical of childhood OSAHS, despite the absence of PSG signs of OSAHS or UARS. These children with primary snoring and several OSAHS symptoms without cortical micro-arousal raise the question of the pathophysiological mechanism or mechanisms of these complications ${ }^{5}$ : what is the "missing link" accounting for the onset of these various disorders? Some authors suggested that the alternating cyclic curves recorded in UARS ${ }^{23}$ might induce the observed cognitive disorders. In adults, isolated airflow restriction exceeding $30 \%$ of sleep time is 
considered "abnormal" and potentially associated with pathologic disorder (Palombini29). Such examples show the sometimes insufficient sensitivity of PSG and current scoring methods for characterizing specific disturbances which are nevertheless manifestly observed in primary snorers.

Exploring and validating the characteristics associated with these clinical disorders could improve the correlation between sleep recording data and clinical symptomatology in sleep-disordered breathing.

\section{Correlation between apnea score and clinical disorder}

The strength of the correlation between $\mathrm{AHI}$ determined on sleep recording and the observed clinical disorders shown by the child or group of children determines the pathologic status of the AHI score. The correlations are relatively well established for groups of children, comparing mean $\mathrm{AHI}$ between children with and without clinical pathology. Likewise, the correlation between $\mathrm{AHI}$ severity and clinical severity can be statistically confirmed in groups of children. Individual correlation between pathologic $\mathrm{AHI}$ and a given morbidity criterion, in contrast, is highly variable, hindering extrapolation of the theoretical set of signs of OSAHS to a given individual child. The strength of the correlation depends on numerous known and unknown individual factors, and on the child's genetic inheritance in particular. For example, the presence of apolipoprotein $\mathrm{E}$ epsilon 4 determines the degree of cognitive impairment associated with OSAHS ${ }^{27}$; consequently, clinical complications in proven sleep-disordered breathing will vary from child to child, depending on the genetic background and the characteristic in question. It would be possible to improve individual complications of sleep-disordered breathing. Information provided to parents by educational software would help them assess the child's symptoms and their impact on psychometric scales. However, certain cognitive-behavioral complications are difficult to assess.

How can an $\mathrm{IO}$ deficit, attention disorder or working memory deficit be identified and attributed to OSAHS in a child with sleep-disordered breathing if there is no baseline assessment? Despite such undeniable difficulties, the clinical impact of apnea needs to be assessed precisely in order to interpret the $\mathrm{AHI}^{2}$, which is in itself too simplistic to be a reliable index of the severity of sleep-disordered breathing and should be only one element among the clinical signs guiding decision-making.

\section{Novel diagnostic examinations in sleep-disordered breathing}

There is a need in current practice for either new diagnostic criteria or new validated signals to improve diagnostic sensitivity, accessibility of examinations and the correlation between polygraphy and clinical morbidity. Current methods can already optimize diagnostic strategy: e.g., measuring the interval between movements during sleep ${ }^{9}$, or the percentage of sleep-time showing restricted inspiration ${ }^{29}$; other methods should also be developed, such as urine assay of biological markers for OSAHS ${ }^{10}$, new non-contact respiratory sensors ${ }^{3}$, phone oximetry ${ }^{11}$ and other connected 
devices yet to come. Measurement of mandibular movement ${ }^{25}$ during sleep could be contributive as it simultaneously assesses oral opening, oral respiration and inspirational effort; validation of this method could improve screening for at-risk groups: children with hyperactivity, enuresis, obesity, educational dif- ficulties, prematurity, familial OSAHS or having undergone adenotonsillectomy. Acloser correlation between respiratory abnormalities and clinical symptoms could emerge from "big data" 30 , allowing better adapted criteria or new polygraphic parameters with better correlation to clinical morbidity.

\section{CONCLUSION}

The current set of respiratory signal abnormalities describes most forms of sleep-disordered breathing but is sometimes insufficient to diagnose certain disorders such as primary snoring. The correlation between $\mathrm{AHI}$ severity and the severity of morbidity does not always hold in individual cases. Waiting times for sleep recording, and especially PSG, can be prohibitive and prevent real public health needs being met; simplified examinations are therefore performed, but tend to underestimate certain forms of sleep-disordered breathing. The physician should be familiar with the method employed and with the patient's clinical history to better interpret results in the light of the sensitivity of the recording device and the patient's individual context. Rather than an oversimplified approach using $\mathrm{AHI}$ alone, a global approach including clinical data will enable progress toward child-centered personalized medicine that is more reliable and better adapted to the complexity of sleep-disordered breathing.

\section{ACKNOWLEDGMENTS}

Thanks to Dr Sarah Hartley for the English translation of the Abstract.
Conflicts of interest: The authors have no conflicts of interest to declare.

\section{REFERENCES}

1. Arens R, Marcus CL. Pathophysiology of upper airway obstruction: a developmental perspective. Sleep 2004;27(5):997-1019.

2. Aurora RN, et al. Practice parameters for the respiratory indications for polysomnography in children. Sleep 2011;34:379-88.

3. Beattie ZT, Hayes TL, Guilleminault C, Hagen CC. Accurate scoring of the apnea-hypopnea index using a simple non-contact breathing sensor. J Sleep Res 2013;22:356-62. 
4. Berry RB, et al. Rules for scoring respiratory events in sleep: update of the 2007 AASM manual for the scoring of sleep and associated events. J Clin Sleep Med 2012;8:597-619.

5. Biggs SN, Nixon GM, Horne RS. The conundrum of primary snoring in children: what are we missing in regards to cognitive and behavioural morbidity? Sleep Medicine Reviews 2014;18:463-75.

6. Brockmann PE, Urshitz MS, Schlaud M, Poets CF. Primary snoring in school children: prevalence and neurocognitive impairments. Sleep and Breathing 2012;16:23-9.

7. Caroll JL. McColly SA, Marcus CL, Curtis S, Loughlin GM. Inability of clinical history to distinguish primary snoring from obstructive sleep apnea in children. Chest 1995;108:610-18.

8. Contencin P, Malorgio E, Noce S, Couloigner V, Vigo A. Questionnaire and nocturnal oxymetry in children with adenotonsillar hypertrophy. Eur Ann Otorhinolaryngol Head Neck Dis 2010;127:137-42.

9. Coussens $\mathrm{S}$, et al. Movement distribution: a new measure of sleep fragmentation in children with upper airway obstruction. Sleep 2014;37:2025-34.

10. De Luca Canto G, Pachêco-Pereira C, Aydinoz S, Major PW, Flores-Mir C, Gozal D. Diagnostic capabil- ity of biological markers in assessment of obstructive sleep apnea: a systematic review and meta-analysis. J Clin Sleep Med 2015;11(1):27-36.

11. Garde A, Dehkordi P, Karlen W, Wensley D, Ansermino JM, Dumont GA. Development of a screening tool for sleep disordered breathing in children using the phone oximeter. Plos One 2014;9:e112959.

12. Guilleminault C, Eldridge F, Simmons FB. Sleep apnea in eight children. Pediatrics 1976;58:28-31.

13. Guilleminault C, Pelayo R, Leger D, Clerck A, Bocian RC. Recognition of sleep-disordered breathing in children. Pediatrics 1996;98:871-82.

14. Guilleminault $C$, Winkle R, Korobkin R, Simmons B. Children and nocturnal snoring: evaluation of the effects of sleep related respiratory resistive load and daytime functioning. Eur J Pediatr 1982;139:165-71.

15. Iber C, Ancoli-Israel S, Cheysson A, Quan SF. The AASM Manuel for the scoring of sleep and associated events: rules, terminology and technical specifications. 1st ed. Westchester, IL: American Academy of Sleep Medicine; 2007.

16. Katz ES, Lutz J, Black C, Marcus CL. Pulse transit time as a measure of arousal and respiratory effort in children with sleep-disordered breathing. Pediatr Res 2003;53:580-8.

17. Kim JH, Guilleminault $\mathrm{C}$. The nasomaxillary complex, the mandible and sleep-disordered breathing. Sleep Breath 2011;15:185-93.

18. Kholer MJ, et al. Differences in the association between obesity and obstructive sleep apnea among children and adolescents. J Clin Sleep Med 2009;5:506-11.

19. Krakow B, Krakow J, Ulibarri VA, Mclver ND. Frequency and accuracy of "RERA" and "RDI" terms in the Journal of Clinical Sleep Medicine from 2006 through 2012. J Clin Sleep Med 2014;10:121-4.

20. Li AM, Au CT, Ho C, Fok TF, Wing YK. Blood pressure is elevated in children with primary snoring. J Pediatr 2009;155(3):362-8.

21. Li AM, Au CT, Chook P, Lam HS, Wing YK. Reduced flow-mediated vasodilatation of brachial artery in children with primary snoring. Int J Cardiol 2013;167:2092-6.

22. Lin $\mathrm{CH}$, Guilleminault C. Current hypopnea scoring criteria underscore pediatric sleep disordered breathing. Sleep Med 2011;12:720-9. 
23. Lopes MC, Guilleminault C. Chronic snoring and sleep in children: a demonstration of sleep disruption. Pediatrics 2006;118:e741-6.

24. Marcus CL. Sleep disordered breathing in children. Am J Resp Crit Care Med 2001;164:16-30.

25. Martinot JB, Denison SJ, Senny FH, Robillard TA, Katwa U, Guenard H. Mandibular movements identify respiratory efforts due to obstructive sleep apnea in a pre-school child. The Open Sleep Journal 2014;7 reprints @benthamscience.net

26. Muzumdar H. Arens R. Diagnostic issues in pediatric obstructive sleep apnea. Proc Am Thorac Soc 2008;5:263-73.

27. Nikodemova M, Finn L, Mignot E, Salzieder N, Peppard PE. Association of sleep disordered breathing and cognitive deficit in APOE epsilon 4 carriers. Sleep 2013;36:873-80.

28. Nixon GM, Hyde M, Biggs SN, Walter LM, Horne RS, Davey MJ. The impacts of recent changes to the respiratory scoring rules in pediatrics. J Clin Sleep Med 2014;10:1217-21.

29. Palombini LO, et al. Inspiratory flow limitation in a normal population of adults in Sao Paulo Brazil. Sleep 2013;36:1663-8.

30. Redline S, Dean D, Sanders MH. Entering the era of "big data": getting our metrics right. Sleep 2013;36:465-9.

31. Recommendation HAS 2012 : Place et conditions de réalisation de la polysomnographie et de la polygraphie respiratoire dans les troubles du sommeil. www. has-sante.fr

32. Section on pediatric pulmonology. Subcommittee on obstructive sleep apnea syndrome. American Academy of Pediatrics. Clinical practice guideline: diagnosis and management of childhood obstructive sleep apnea syndrome. Pediatrics 2002;109:704-12.

33. Scholle S, Wiater A, Scholle HC. Normative values of polysomnographic parameters in childhood and adolescence: arousal events. Sleep Med 2012;13:243- 51.

34. Spryut K, Gozal D. Screening of pediatric sleep-disor- dered breathing: a proposed unbiased discriminative set of questions using clinical severity scales. Chest 2012;142:1508-15.

35. Tan HL, Gozal D, Ramirez HM, Bandla HP, Kheirandish- Gozal L. Overnight polysomnography versus respiratory polygraphy in the diagnosis of pediatric obstructive sleep apnea. Sleep 2014;37:255-60.

36. Uliel S, Tauman R, Greenfeld M, Sivan Y. Normal polysomnographic respiratory values in children and adolescents. Chest 2004;125:872-8.

37. Villa MP, et al. Sleep clinical record: an aid to rapid and accurate diagnosis of paediatric sleep disordered breathing. Eur Respir J 2013;41:1355-61.

38. Weinstock TG, et al. Sleep 2014;37:261-9.

39. Zicari AM, et al. Serum resistin in children with primary snoring. Int J Immunopathol Pharmacol 2014;27(3):449- 54. 